EPJ Web of Conferences 45, 01024 (2013)

DOI: $10.1051 /$ epjconf/20134501024

(c) Owned by the authors, published by EDP Sciences, 2013

\title{
Re-evaluation of experimental data on the second virial coefficient for steam and development of its analytical representation as a function of the internal energy
}

\author{
M. Duška ${ }^{11}$ DQGJ. Hrubý ${ }^{1}$ \\ ${ }^{1}$ Institute of Thermomechanics AS CR, v.v.i, Dolejškova 5, Prague 8, CZ-18200, Czech Republic
}

\begin{abstract}
A re-evaluation of the second virial coefficient of steam is presented in the paper. The work is a part of broader effort to develop a formulation of the properties of dry and metastable steam suitable for CFD computations. The re-evaluation follows up previous work by Harvey and Lemmon [1], however with a special care for the lower temperature region close to the triple point and including more experimental data. The second virial coefficient was evaluated from volumetric (pvT) data, calorimetric measurements for saturated vapor, steam expansion experiments (measurements of the Joule-Thomson coefficient and the isothermal throttling coefficient) and measurements of the speed of sound. To accurately evaluate the uncertainty of calorimetric measurements, the uncertainty of the temperature derivative of the saturation pressure was determined based on refitting of the IAPWS saturation pressure formula to the experimental data. In the second step, the evaluated data and their uncertainties were used to develop an analytical formula to compute the second virial coefficient as function of internal energy in a range corresponding to the ideal-gas temperatures from $273.16 \mathrm{~K}$ to $1073.15 \mathrm{~K}$. The choice of internal energy and density as independent variables is required for the CFD computations to avoid time-consuming iterations.
\end{abstract}

\section{Introduction}

A new formulation of thermodynamic property of steam and water designed for CFD computation [2] describes residual entropy as a function of density and internal energy in the form of a virial equation. Therefore the virial coefficients as the function of internal energy instead of thermodynamic temperature are required.

Moreover there is another need for re-evaluation of the virial coefficients. The accuracy of virial coefficients is critical to proper description of the metastable region because of the multiplicative affect of virial coefficients error on the thermodynamic property accuracy (eg. compressibility factor from equation (1)), increasing progressively with density.

The lower temperature region is usually out of interest because of a little difference of steam properties from the ideal gas ones on the other hand, the metastable region connected with the low temperature region in direction of increasing density lies in centre of low-pressure turbine interests [3]. The re-evaluation of second virial coefficient is therefore focusing on correct interpretation of its uncertainty with main interest in the lower temperature region where measured data are scarce.

\section{Methodologies}

The virial equation of state is a fundamental equation describing thermodynamic properties of gases at low to moderate densities

$$
\frac{p(T, \rho)}{\rho R T}=Z(T, \rho)=1+B(T) \rho+C(T) \rho^{2}+\ldots,
$$

where $p$ is the pressure, $T$ the thermodynamic temperature, $\rho$ the density, $R$ the gas constant, $Z$ the compressibility factor and $B$ and $C$ are the second and the third virial coefficients, respectively. This formulation can be transformed to describe various thermodynamic properties.

The virial coefficients can be determined for isothermal data sets separately, or by fitting virial equation and functions of virial coefficients at once on whole set of measured data. The first method was chosen to determined second virial coefficient because of its simplicity and because of simpler elimination of a mutual influence the virial coefficients have on each other during the fitting procedure. 


\subsection{Determination of the second virial coefficient}

There are two approaches based on different kinds of available measured data to determined second virial coefficient. Thermodynamic properties on the vapourliquid boundary can be used to determine only the second virial coefficient. One measured point for every temperature can together with a theoretical point of ideal gas property form only linear relationship. The equation (1) reduce to

$$
Z\left(T, \rho^{\prime \prime}\right)=1+B(T) \rho^{\prime \prime}+\Delta z,
$$

where $\Delta z$ is the correction for ignoring higher order virial coefficients. The correction based on IAPWS95 multiparameter equation of state [4] $(\Delta z$ is for particular temperature calculated from equation (2) while all other variables were based on IAPWS95) should be added to its uncertainty.

$$
\Delta B=\sqrt{\sum_{i=1}^{l}\left(\frac{\partial B}{\partial t_{i}}\right)^{2} \sigma_{i}^{2}}+\left|\frac{\Delta z}{\rho^{\prime \prime}}\right|,
$$

where $t$ are the measured thermodynamic properties and their uncertainty $\sigma$.

The second method is based on isothermal data of thermodynamic property of steam. The equation (1) is directly fitted on experimental data. It is important to decide an order of the virial equation or extend of the experimental data used in fitting procedure to satisfied preconditions of regression method described later.

A third order virial equation was usually used or second order one if it describes data correctly. To ensure that select order of virial equation describes data set correctly without significant influence of higher virial coefficients, the regression residuals were checked if they do not exceed the standard deviation of measurement uncertainty. If the third order virial equation failed this test the data set was reduced on a side of high density or pressure respectively and checked again.

\subsection{Weighted linear regression}

Weighted linear regression was applied to fined regression coefficients in terms of the column vector of regression parameters

$$
\mathbf{a}=\left(\mathbf{X}^{\prime} \Sigma^{-1} \mathbf{X}\right)^{-1} \mathbf{X}^{\prime} \Sigma^{-1} \mathbf{y}
$$

where $\mathbf{y}$ is column vector of the variables, $\mathbf{X}$ the matrix with known regression coefficients and $\boldsymbol{\Sigma}$ the covariance weight matrix. There is several condition considered to be meet by measured data and regression function. The regression residuals are fully explained by uncertainty of measurement and uncertainty of measurement is random error only with known standard deviation. Then the weighted matrix is diagonal matrix with elements

$$
\sigma_{\Delta i}^{2}=\sum_{l=1}^{3}\left(\frac{\partial \Delta_{i}}{\partial t_{l i}}\right)^{2} \sigma_{l i}^{2}
$$

where $t$ are the measured thermodynamic properties and their uncertainty $\sigma$ and

$$
\Delta_{i}=y_{i}-\sum_{j=1}^{m} x_{i j} a_{j} .
$$

Then a covariance matrix of $\mathbf{a}$ is

$$
V(a)=\left(X^{\prime} \Sigma^{-1} X\right)^{-1}
$$

and a confidence interval of the regression parameter is

$$
a_{i} \pm \sqrt{V_{i, i}(a) \chi_{\alpha}^{2}(m)},
$$

where $\chi_{\alpha}^{2}$ is Chi-square inverse cumulative distribution function with $m$ degrees of freedom and probability $\alpha$ is set to be equal to probability of standard deviation.

\section{The second virial coefficient from thermodynamic properties on the vapour-liquid boundary}

\subsection{Calorimetric property of vaporization}

Three special calorimetric properties were measured by Osborne et al. [5] and [6] from the triple to the critical points. A special calorimetric property $\gamma$ can be used to calculate the saturated density with the Clapeyron equation

$$
\rho^{\prime \prime}=\frac{T}{\gamma} \frac{\mathrm{d} p_{\mathrm{s}}}{\mathrm{d} T},
$$

where $p_{\mathrm{s}}$ is the saturated pressure. Then the second virial coefficient can be calculated

$$
B(T)=\frac{p_{\mathrm{s}}}{\left(\frac{T}{\gamma} \frac{\mathrm{d} p_{\mathrm{s}}}{\mathrm{d} T}\right)^{2} T R}-\frac{1+\Delta z}{\frac{T}{\gamma} \frac{\mathrm{d} p_{\mathrm{s}}}{\mathrm{d} T}} .
$$

The main challenge is to determine the uncertainty of the virial coefficient. The uncertainty of variables (saturated pressure and its derivative and calorimetric property $\gamma$ ) should be determined. There is also a possibility of their interdependence which should be considered.

The regression of saturated vapour pressure and its derivative was published in [7] and in details in [8] (at ITS-90 temperature scale in [9]). There were also published the percentage uncertainties in vapour pressure [4] for IAPWS95 formulation based on the regression but no data for the uncertainty of the saturated vapour pressure derivative are available. The uncertainty of the 
saturated pressure derivative was therefore developed and given in appendix A.1.

The development of the uncertainty of the saturated pressure derivative is based on assumptions described in section 2.2 where only random error is considered. A possible systematic error of measurement leads to higher uncertainties of the regression.

On a purely random error an uncertainty of special calorimetric property $\gamma$ is biased. The uncertainty was calculated as a standard deviation of measured data.

A statistical meaning of the uncertainty of vapour pressure published in [4] is less clear but was used in this study for its recognition and as considerably conservative estimate compare to the pervious uncertainties.

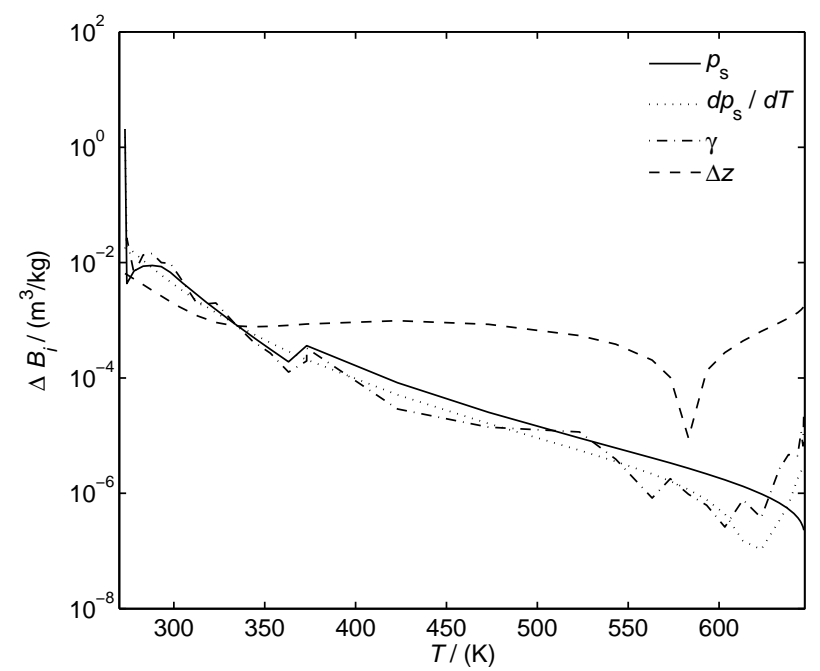

Fig. 1. Components of second virial coefficient uncertainty for the calorimetric measurement.

The influence of the uncertainties on the uncertainty of the second virial coefficient can be seen from figure 1 . The results are surprisingly similar. The uncertainties of saturated pressure and its derivative should be somehow correlated on the other side the uncertainty of special calorimetric property $\gamma$ is probably uncorrelated on the pressure measurement. Despite possible correlation all three uncertainties were used to determine the uncertainty of second virial coefficient.

The correction for neglecting higher order virial coefficients has a dominant role for higher temperatures. A local minimum which can be seen in figure 1 is caused by change between the positive and the negative effect it has on the second virial coefficient.

The selected results can be seen from table 1 .

Table 1. Selected results of the second virial coefficient and its uncertainty from calorimetric measurement.

\begin{tabular}{|c|c|c|c|}
\hline $\boldsymbol{u}(\mathbf{J} / \mathbf{k g})$ & $\boldsymbol{T}(\mathbf{K})$ & $\boldsymbol{B}\left(\mathbf{c m}^{\mathbf{3}} / \mathbf{m o l}\right)$ & $\boldsymbol{\Delta B}\left(\mathbf{c m}^{\mathbf{3}} / \mathbf{m o l}\right)$ \\
\hline 2417375 & 303.134 & -1203 & 181 \\
\hline 2431424 & 313.13 & -969 & 92 \\
\hline 2445503 & 323.127 & -834 & 68 \\
\hline 2459613 & 333.124 & -722 & 42 \\
\hline
\end{tabular}

\begin{tabular}{|l|l|l|l|}
\hline 2473760 & 343.124 & -630 & 30 \\
\hline 2487942 & 353.123 & -557 & 25 \\
\hline 2502164 & 363.123 & -496 & 21 \\
\hline 2516427 & 373.124 & -453 & 24 \\
\hline 2516427 & 373.124 & -447 & 25 \\
\hline 2588422 & 423.134 & -287 & 20 \\
\hline 2661692 & 473.153 & -201 & 16 \\
\hline 2736361 & 523.171 & -148 & 10 \\
\hline 2766642 & 543.178 & -133 & 7 \\
\hline 2797161 & 563.182 & -120 & 4 \\
\hline 2812513 & 573.184 & -114 & 2 \\
\hline 2827926 & 583.185 & -108 & 0.2 \\
\hline 2843402 & 593.186 & -103 & 2 \\
\hline 2858938 & 603.186 & -99 & 5 \\
\hline 2874540 & 613.187 & -94 & 8 \\
\hline
\end{tabular}

\subsection{Speed of sound}

The speed of sound was measured in saturated and under saturated steam by Novikov and Avdonin [10]. The measurement for the saturated vapor would be a valuable source because it was carried out in low temperatures from $273 \mathrm{~K}$ to $583 \mathrm{~K}$, the total error of speed of sound measurement was within $1 \%$ limit. Therefore this set of measurement was selected to analyze its suitability to determine the second virial coefficient.

The relation between the speed of sound and the second virial coefficient can be described according to [11] by the flowing equation

$$
w^{2}=A_{0}+A_{1} p+A_{2} p^{2}+\Delta w^{2},
$$

where

$$
A_{0}=k_{0} R T,
$$

$$
\begin{gathered}
A_{1}=k_{0}\left[2 B+2 B_{t}\left(k_{0}-1\right)+\frac{B_{t t}\left(k_{0}-1\right)^{2}}{k_{0}}\right], \\
A_{2}=\frac{a_{2 B}}{R T},
\end{gathered}
$$

$\Delta w$ is the correction for ignoring higher order virial coefficients described in appendix A.2 and

$$
k_{0}=\frac{c_{p}^{0}}{c_{p}^{0}-R},
$$




$$
\begin{aligned}
a_{2 B}= & -\left(k_{0}+1\right) B^{2}+\left(k_{0}-1\right)\left(2 k_{0}-1\right)^{2} B_{t}^{2}+\left(k_{0}-1\right)^{3} B_{t t}^{2} \\
& +\left(k_{0}-1\right)^{2}\left[2 B B_{t}+B B_{t t}+\left(4 k_{0}-2\right) B_{t} B_{t t}\right]
\end{aligned}
$$$$
B_{t}=T \frac{\partial B}{\partial T}
$$$$
B_{t t}=T^{2} \frac{\partial^{2} B}{\partial T^{2}} .
$$

The valuable second virial coefficient can be determined only if the real thermodynamic property differs from the ideal significantly compared to its uncertainty of measurement. On the other hand the correction for neglecting higher order virial coefficients should not increase the overall uncertainty significantly. Figure 2 compares the measurement and its uncertainty with the ideal gas speed of sound, speed of sound calculated from IAPWS 95 and one calculated from equation (11) where the correction is set to be zero and the second virial coefficient is calculated from IAPWS 95.

As can be seen from the figure the measured data are in a very good agreement with the IAPWS 95 formulation. But uncertainty of measurement is too high compared to the difference between the real and the ideal property for much of the measurements at least up to 500 $\mathrm{K}$. The temperature of $500 \mathrm{~K}$ is also a region where the correction for neglecting higher orders virial coefficients starts to be the dominant source of uncertainty.

Based on the preliminary analyses the speed of sound measurement, we found that it cannot be used for determining accurate second virial coefficient because of the too high uncertainty of the measurement.

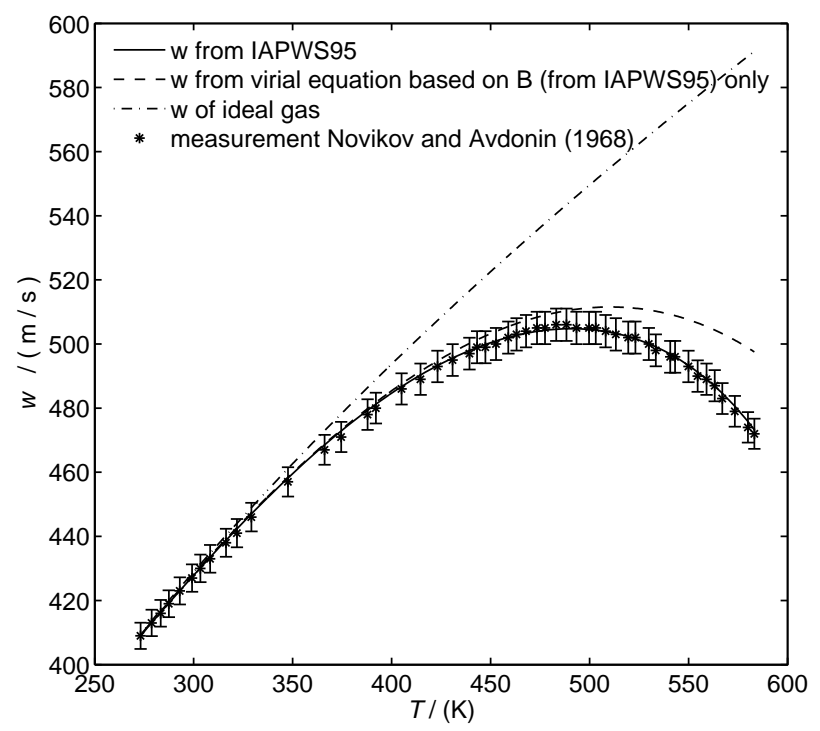

Fig. 2. Speed of sound measured data with context of mathematical models.

\section{The second virial coefficient from isothermal data}

\subsection{PVT data}

Vukalovich et al. [12] in 1967 published virial equation of state for steam at temperatures above $500{ }^{\circ} \mathrm{C}$. They was also presented a table of the second and third virial coefficients based on a previous volumetric (pvT) measurement (for temperatures from $400{ }^{\circ} \mathrm{C}$ to $900^{\circ} \mathrm{C}$ ) measured by the authors. But to determine the uncertainty of the coefficient, a re-evaluation was necessary. The original data of volumetric (pvT) measurement was published in [13] and [14]. The data consist of 23 isothermal measurements of the volume for pressure from 4.5 $\mathrm{MPa}$ to $118.4 \mathrm{MPa}$.

The uncertainty of temperature was 0.1 and $0.2 \mathrm{~K}$ and the uncertainty of volume 0.2 and $0.25 \%$ for data measured up to $700^{\circ} \mathrm{C}$ and above, respectively. Uncertainty of the pressure measurement was $0.05 \%$ for every measurement.

To determine the second virial coefficient and its uncertainty from the measurement, the method described in section 2 was applied. The fitted equation was slightly modified equation (1)

$$
\frac{p v}{R T}-1=+\frac{B}{v}+\frac{C}{v^{2}} .
$$

The regression curves for all isotherms and the data the regression is based on are presented in figure 3 . The resulting second virial coefficients and their uncertainty are given in table 2 .

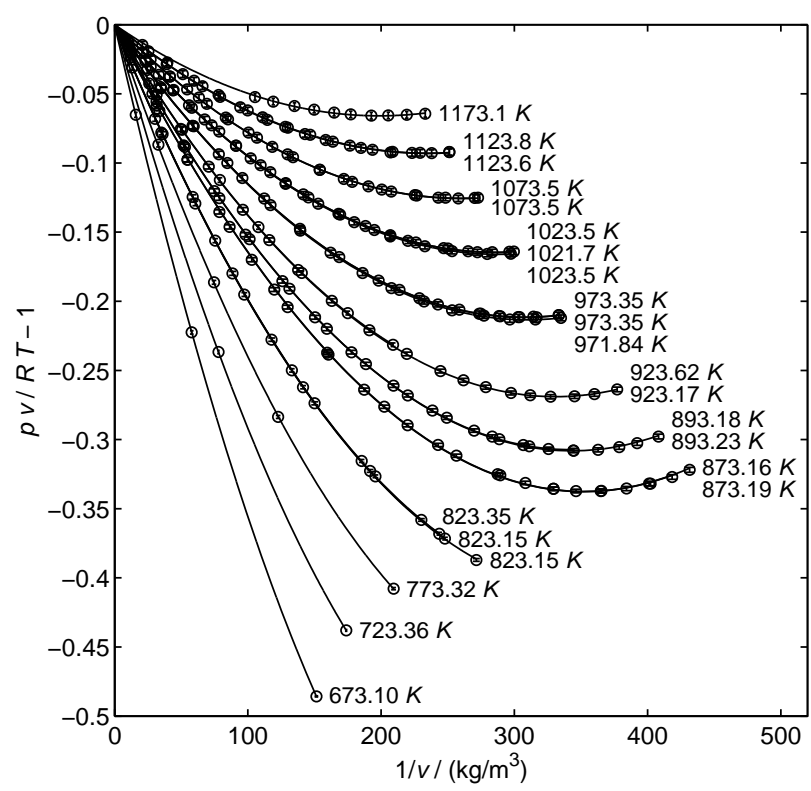

Fig. 3. Regression curves representing PVT measurement [13] and [14]. 
Table 2. Second virial coefficient and its uncertainty from PVT measurement [13] and [14].

\begin{tabular}{|c|c|c|c|c|}
\hline $\boldsymbol{u}(\mathbf{J} / \mathbf{k g})$ & $\boldsymbol{T}(\mathbf{K})$ & $\begin{array}{c}\text { Number of } \\
\text { measured/ } \\
\text { selected } \\
\text { points }\end{array}$ & $\begin{array}{c}\boldsymbol{B} \\
\left(\mathbf{c m}^{\mathbf{3}} / \mathbf{m}\right. \\
\mathbf{o l})\end{array}$ & $\begin{array}{c}\Delta \boldsymbol{B} \\
\left(\mathbf{c m}^{3} / \mathbf{m}\right. \\
\mathbf{o l})\end{array}$
\end{tabular}$\quad$ and from Joule-Thomson coefficient $\delta_{\mathrm{h}}$

The fitted equation for isothermal throttling coefficient is

$$
\delta_{\mathrm{T}}=\left(B-T \frac{\mathrm{d} B}{\mathrm{~d} T}\right)+\left(C^{\prime \prime}-T \frac{\mathrm{d} C^{\prime \prime}}{\mathrm{d} T}\right) p+\left(D^{\prime \prime}-T \frac{\mathrm{d} D^{\prime \prime}}{\mathrm{d} T}\right) p^{2},
$$

where the third element of the right side of the equation was used for regression only if necessary and $C^{\prime \prime}=\left(C-B^{2}\right) / R T$.

The equation for regression of Joule-Thomson coefficient is

$$
-\delta_{\mathrm{h}} c_{\mathrm{p}}^{0}=\left(B-T \frac{\mathrm{d} B}{\mathrm{~d} T}\right)+\frac{C^{\prime \prime}-T \mathrm{~d} C^{\prime \prime} / \mathrm{d} T}{c_{\mathrm{p}} / c_{\mathrm{p}}^{0}} p
$$

\subsubsection{Temperature corrections}

The sets of measurement were carried out very close to a mean temperature (usually deviating less than by $1 \mathrm{~K}$ ) but their variation were too large compared to the uncertainty of the temperature measurement. Corrections of the coefficients to the mean temperature $T_{m}$ is

$$
\begin{aligned}
\Delta \delta^{(i)}\left(T_{m}-T_{j}\right) & =\left[b^{(i)}\left(T_{m}\right)+c^{(i)}\left(T_{m}\right) p_{j}\right] \\
& -\left[b^{(i)}\left(T_{j}\right)+c^{(i)}\left(T_{j}\right) p_{j}\right]
\end{aligned}
$$

where $\delta=\delta_{\mathrm{T}}$ for the isothermal throttling coefficient and $\delta=\delta_{\mathrm{h}} c_{\mathrm{p}}^{0}$ for the Joule-Thomson coefficient. The temperature dependent functions $b$ and $c$ represent the first and the second parameter of equations (22) and (23). At first, the parameters $b$ and $c$ can be found for every isothermal data set without temperature correction. The functions are in the following forms for the isothermal throttling coefficient

$$
b(T)=\sum_{i=0}^{3} \frac{b_{i}}{T^{i}}, \quad c(T)=\sum_{i=0}^{4} \frac{c_{i}}{T^{i}},
$$

The last evaluated source of data is a measurement of the isothermal throttling coefficient and the Joule-Thomson coefficient carried out by Ertle [15]. The isothermal throttling coefficient was measured from $622 \mathrm{~K}$ to $1073 \mathrm{~K}$ with uncertainty of $1.4 \%$ and the Joule-Thomson coefficient from $431 \mathrm{~K}$ to $623 \mathrm{~K}$ with uncertainty of $1.7 \%$ and then to $1073 \mathrm{~K}$ with uncertainty of $1.4 \%$.

The second virial coefficient and its derivative property $B-T \mathrm{~d} B / \mathrm{d} T$ can be estimated from isothermal throttling coefficient $\delta_{\mathrm{T}}$

)


until the parameters $b$ and $c$ do not change more than by $0.001 \%$.

\subsubsection{Second virial coefficient}

The regression parameter $a_{1}=B-T \mathrm{~d} B / \mathrm{d} T$ can be calculated from the corrected coefficients. Figure 4 shows corrected isothermal throttling coefficient data with regression functions and their uncertainties. The only regression for temperature $622.789 \mathrm{~K}$ was based on all three parameters of equation (22). The figure 5 presents the Joule-Thomson coefficient data and the regression.

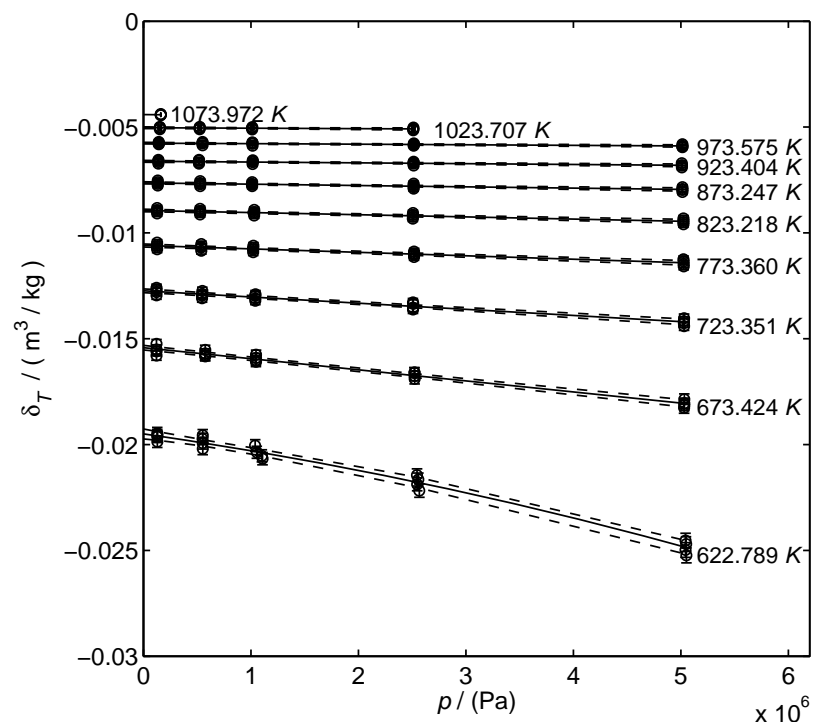

Fig. 4. Regression curves representing the isothermal throttling coefficient measurement.

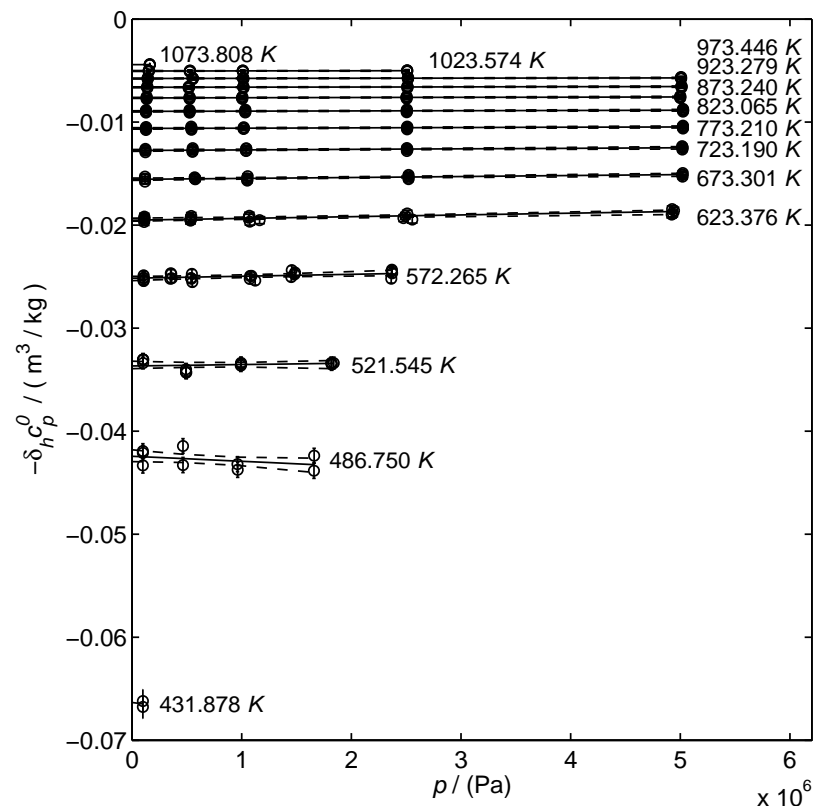

Fig. 5. Regression curves representing the Joule-Thomson coefficient measurement.

If there was not enough data for a particular temperature to calculate any regression (for isothermal throttling coefficient temperature $1073.972 \mathrm{~K}$ and for Joule-Thomson coefficient temperatures $431.878 \mathrm{~K}$ and
$1073.808 \mathrm{~K})$, an intercept a1 is calculated from the slope based on functions $c$ (equation (25) or (26)) and mean point of the coefficients and pressure. Second virial coefficient and its derivative based on this procedure is just informative.

The resulting second virial coefficient and its derivative properties $a_{1}=B-T \mathrm{~d} B / \mathrm{d} T$ are summarised in table 3 for the isothermal throttling coefficient and in table 4 for the Joule-Thomson coefficient together with their uncertainties. An uncertainty of $a_{1}=B-T \mathrm{~d} B / \mathrm{d} T$, calculated beside a lack of sufficient number of measured data (italic type), was based on guess extrapolation of relative uncertainties.

Table 4. Second virial coefficient and its uncertainty from the isothermal throttling coefficient measurement.

\begin{tabular}{|c|c|c|c|c|}
\hline $\boldsymbol{u}(\mathbf{J} / \mathbf{k g})$ & $\boldsymbol{T}(\mathbf{K})$ & $\begin{array}{c}\boldsymbol{a}_{\mathbf{1}} \\
\left(\mathbf{c m}^{\mathbf{3}} / \mathbf{m o l}\right)\end{array}$ & $\begin{array}{c}\Delta \boldsymbol{a}_{\mathbf{1}} \\
\left(\mathbf{c m}^{\mathbf{3}} / \mathbf{m o l}\right)\end{array}$ & $\begin{array}{c}\Delta \boldsymbol{a}_{\mathbf{1}} \\
(\mathbf{\%})\end{array}$ \\
\hline 2889580 & 622.789 & -351.3 & 4.2 & 1.2 \\
\hline 2969871 & 673.424 & -277.8 & 2.0 & 0.7 \\
\hline 3050690 & 723.351 & -229.4 & 1.6 & 0.7 \\
\hline 3133324 & 773.360 & -190.9 & 1.3 & 0.7 \\
\hline 3217420 & 823.218 & -161.0 & 1.1 & 0.7 \\
\hline 3303546 & 873.247 & -137.6 & 0.9 & 0.7 \\
\hline 3391668 & 923.404 & -119.2 & 0.8 & 0.7 \\
\hline 3481601 & 973.575 & -103.8 & 0.7 & 0.7 \\
\hline 3573253 & 1023.707 & -90.7 & 0.7 & 0.8 \\
\hline 3666931 & 1073.972 & -79.5 & 1.1 & 1.4 \\
\hline
\end{tabular}

Table 3. Second virial coefficient and its uncertainty from the Joule-Thomson coefficient measurement.

\begin{tabular}{|c|c|c|c|c|}
\hline $\boldsymbol{u}(\mathbf{J} / \mathbf{k g})$ & $\boldsymbol{T}(\mathbf{K})$ & $\begin{array}{c}\boldsymbol{a}_{\mathbf{1}} \\
\left(\mathbf{c m}^{3} / \mathbf{m o l}\right)\end{array}$ & $\begin{array}{c}\Delta \boldsymbol{a}_{\mathbf{1}} \\
\left(\mathbf{c m}^{\mathbf{3}} / \mathbf{m o l}\right)\end{array}$ & $\begin{array}{c}\Delta \boldsymbol{a}_{\mathbf{1}} \\
(\mathbf{\%})\end{array}$ \\
\hline 2601134 & 431.878 & -1195.2 & 20.3 & 1.7 \\
\hline 2681846 & 486.750 & -763.5 & 10.4 & 1.4 \\
\hline 2733910 & 521.545 & -604.8 & 6.5 & 1.1 \\
\hline 2811100 & 572.265 & -453.7 & 3.7 & 0.8 \\
\hline 2890500 & 623.376 & -350.9 & 2.7 & 0.8 \\
\hline 2969673 & 673.301 & -280.0 & 1.9 & 0.7 \\
\hline 3050427 & 723.190 & -229.7 & 1.6 & 0.7 \\
\hline 3133074 & 773.210 & -191.2 & 1.3 & 0.7 \\
\hline 3217159 & 823.065 & -161.4 & 1.1 & 0.7 \\
\hline 3303535 & 873.240 & -137.8 & 0.9 & 0.7 \\
\hline 3391445 & 923.279 & -119.3 & 0.8 & 0.7 \\
\hline
\end{tabular}


Table 3. - continuing

\begin{tabular}{|l|c|c|c|c|}
\hline 3481368 & 973.446 & -104.0 & 0.7 & 0.7 \\
\hline 3573007 & 1023.574 & -90.8 & 0.7 & 0.8 \\
\hline 3666623 & 1073.808 & -79.5 & 1.1 & 1.4 \\
\hline
\end{tabular}

\section{Development of an analytical representation of the second virial coefficient as a function of the internal energy}

The re-evaluation will be used for developing a new analytical representation of second virial coefficient as a function of the internal energy. As the earlier step a following function was developed

$$
B(u)=\frac{b_{1}}{\rho_{\mathrm{c}}}+\frac{b_{2}}{\rho_{\mathrm{c}} \xi^{2}}+\frac{b_{3}}{\rho_{\mathrm{c}} \xi^{4}}
$$

where

$$
\begin{gathered}
\xi=\beta_{2}-\frac{1}{\frac{u}{R T_{c}}-\beta_{1}}, \\
b_{1}=0.5125, \quad b_{2}=-0.00999, \quad b_{3}=3.22 \times 10^{-7}
\end{gathered}
$$

and

$$
\beta_{1}=3.0136, \quad \beta_{2}=0.21825 \text {. }
$$

Parameters $b_{1}, b_{2}, b_{3}$ and $\beta_{1}, \beta_{2}$ were fitted to the correlation by Harvey and Lemmon [1]. Deviation of the developed function do not exceed the function [1] by more the $\mathrm{P} / \mathrm{o}$ within temperature range $253.15 \mathrm{~K}$ to $1073.15 \mathrm{~K}$.

Developed model is compared with the re-evaluated data, previously published virial coefficients by Adulagatov et al. [16], Eubank et al. [17], Hendl et al. [18], Kell et al. [19] and [20] and with previous models: IAPWS95 [4], Hill and MacMillan [21] and Le Fevre et al. [22] as can be seen from figure 6 for lower internal energy of ideal gas and from figure 7 for higher one.

The presented model can be compared to the $a_{1}=B-T \mathrm{~d} B / \mathrm{d} T$ data when derivative $\mathrm{d} B(u) / \mathrm{d} T$ is defined as

$$
\frac{\mathrm{d} B(u)}{\mathrm{d} T}=\frac{\mathrm{d} B(u)}{\mathrm{d} u}\left(\frac{\mathrm{d} u}{\mathrm{~d} T}\right)_{\rho=0},
$$

where $\left(\frac{\mathrm{d} u}{\mathrm{~d} T}\right)_{\rho=0}$ is the ideal-gas isochoric heat capacity as a function of the internal energy presented in [3]. In figure 8 the model is compared to the previous models and a data measured by McGlashan and Wormald [23].

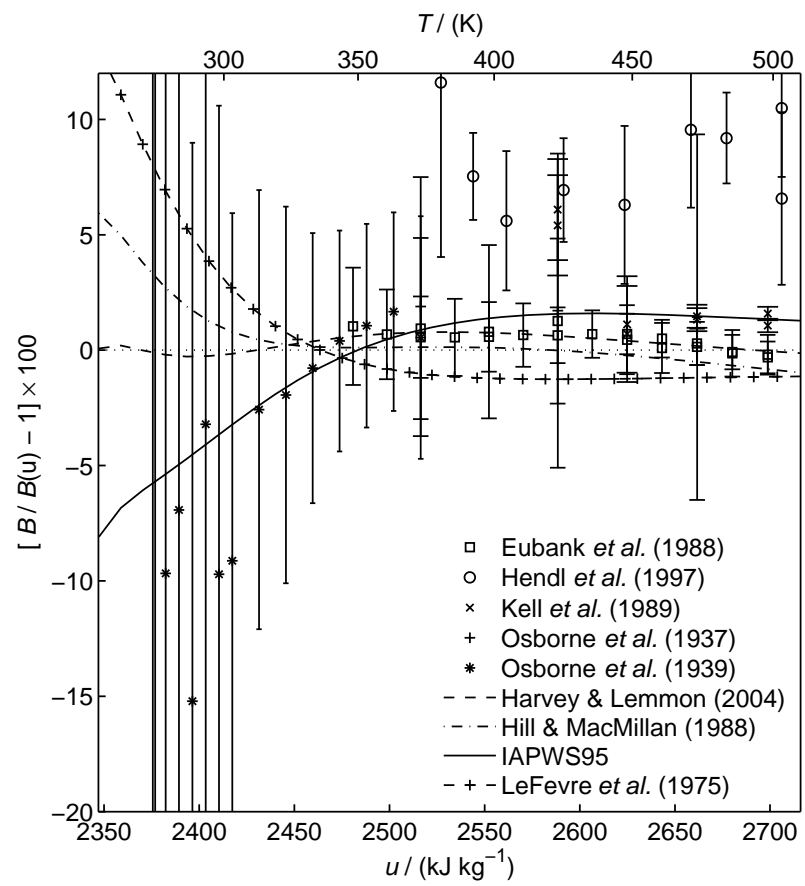

Fig. 6. : Percentage deviations of the second virial coefficient from various sources from the presented model (from 2350 to $2700 \mathrm{~kJ} \mathrm{~kg}^{-1}$ ).

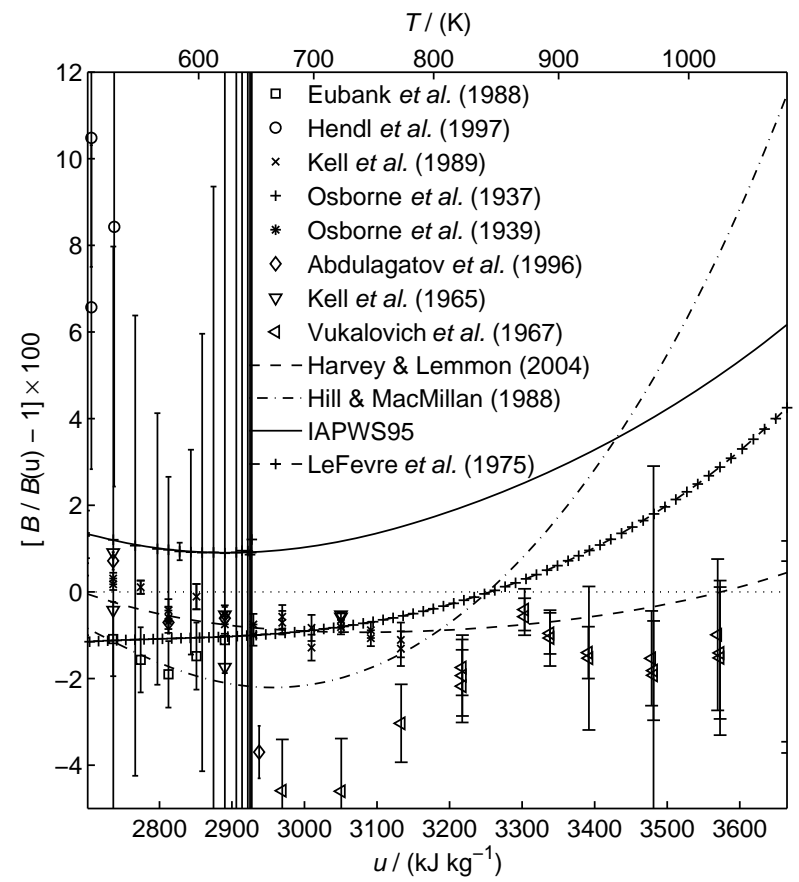

Fig. 7. Percentage deviations of the second virial coefficient from various sources from the presented model (from 2700 to $3650 \mathrm{~kJ} \mathrm{~kg}^{-1}$ ). 


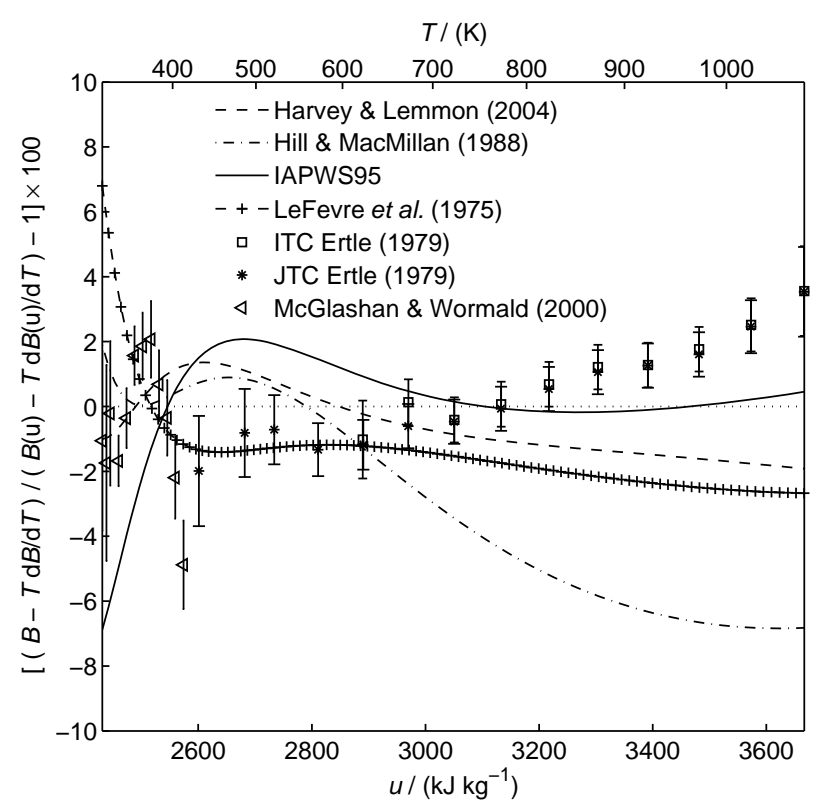

Fig. 8. Percentage deviations of the $a_{1}=B(u)-T d B(u) / d T$ from various sources from the presented model.

\section{Discussions and conclusions}

A data set was prepared for development of a new second virial coefficient formulation. There is no reliable data for temperatures under $300 \mathrm{~K}$. The coefficients based on the Osborne [6] calorimetric measurement have an unacceptably height uncertainty in this region. In the region from $300 \mathrm{~K}$ to $350 \mathrm{~K}$ there are only the measurements by Osborn [6] and by McGlashan and Wormald [23] available. Based on the uncertainty of the measurements, the latter is preferred for fitting the new formulation. From $350 \mathrm{~K}$ to $500 \mathrm{~K}$ the data sets are consistent with except of Hendl et al. [17] measurement. In the following region to $800 \mathrm{~K}$ the data sets are rather inconsistent and difficult to assess. The data in the region of the highest temperatures are also scarce. The data of Vukalovich et al. [13], [14] were re-evaluated to determine the uncertainty of measurement but the results are less consistent with trends of current models.

The speed of sound measurement was tested to be used for determining the second virial coefficient. The results indicated that a significant improvement of the uncertainty of measurement would be needed to make measurement of the thermodynamic property useful for this purpose.

The regression approach applied in the study avoided systematic errors of measurements. In most of the cases it is hard to split correctly the systematic error from the random error and error of the regression model which is also highly correlated as a systematic error. This simplification has strong influence on uncertainty of regression and detailed study of this phenomenon would be very useful.

\section{Acknowledgment}

We gratefully acknowledge support by grants GAAS CR IAA200760905, GA CR 101/09/1633, GAP101/11/1593 and MSMT LA09011.

\section{References}

1. A.H. Harvey, E.W. Lemmon, J. Phys. Chem, Ref. Data 33, 369 (2004)

2. J. Hrubý, International Conference Experimental Fluid Mechanics 2011, 177 (2011)

3. J. Hrubý, J. Pátek, M. Duška Baumann Centenary Wet Steam Conference 10-11 September 2012, (2012)

4. W. Wagner, A. Pruß, J. Phys. Chem. Ref. Data 31, 387 (2002)

5. N.S. Osborne, H.F. Stimson, D.C. Ginnings, J. Res. Natl. Bur. Stand. 18, 389 (1937)

6. N.S. Osborne, H.F. Stimson, D.C. Ginnings, J. Res. Natl. Bur. Stand. 23, 197 (1939)

7. A. Saul, W. Wagner, J. Phys. Chem, Ref. Data 14, 893 (1987)

8. W. Wagner, A. Saul, Proceedings of the 10th international Conferenceon the Properties of Steam 1, 199 (1984)

9. W. Wagner, A. Pruß, J. Phys. Chem, Ref. Data 22, 783 (1993)

10. I. I. Novikov, V. I. Avdonin, Report at the 7 th International Conference of the Properties of Steam (1968)

11. G. Esper, W. Lemming, W. Beckermann, F. Kohler, Fluid Phase Equilibria 105, 173 (1995)

12. M.P. Vukalovich, M.S. Trakhtengerts, G.A. Spiridonov, Thermal Engineering 14, 86 (1967)

13. M.P. Vukalovich, V.N. Zubarev, A.A. Aleksandrov, Teploenergetika 1, 49 (1962)

14. M.P. Vukalovich, V.N. Zubarev, A.A. Aleksandrov, Teploenergetika 10, 79 (1961)

15. S. Ertle, Dissertation, Technische Universität München, Germany, (1979)

16. I.M.Abdulagatov, A.R. Bazaev, R.K. Gasanov, A.E. Ramazanova, J. Chem. Thermodynamics 28, 1037 (1996)

17. P.T.Eubank, L.L. Joffrion, M.R. Patel, W. Warowny, J. Chem. Thermodynamics 20, 1009 (1988)

18. H. Hendl, E. Bich, E. Vogel, J. Chem. Thermodynamics. 29, 765 (1997)

19. G.S. Kell, G.E. McLaurin, E. Whalley, Proc. R. Soc. Lond. 425, 49 (1989)

20. G.S. Kell, G.E. McLaurin, E. Whalley, 3th Symposium on thermophysical properties: advances in thermophysical properties at extreme temperatures and pressures, 104 (1965)

21. P.G. Hill, R.D.Ch. MacMillan, Ind. Eng. Chem. Res. 27, 874 (1988)

22. E.J. Le Fevre, M.R. Nightingale, J.W. Rose, J. Mech. Eng. Sci. 17, 243 (1975)

23. M.L. McGlashan, C.J. Wormald, J. Chem. Thermodynamics 32, 1489 (2000) 


\section{Appendices}

\section{A.1 Uncertainty of the derivation of the saturation pressure}

To develop uncertainty of the regression of saturation pressure derivative, a couple of assumptions were applied. Some of them were mentioned above in the description of regression procedure

$\ln \left(\frac{p_{\mathrm{s}}}{p_{\mathrm{c}}}\right)=\frac{T c}{T}\left(a_{1} \vartheta+a_{2} \vartheta^{1.5}+a_{3} \vartheta^{3}+a_{4} \vartheta^{3.5}+a_{5} \vartheta^{4}+a_{6} \vartheta^{7.5}\right)$

The six coefficients of saturation vapour pressure regression function were originally selected from twentyone "bank of terms" using the evolutionary optimization method [12]. But after the setting the coefficients regression the weighted linear regression can be used to refitting the regression and to determined covariance matrix of regression coefficients (equations (4) - (7)).

According to [11] the original regression is based on measurements of [5], [6], [8], [1], [2] and [3] and uncertainty of measurement to determine the weight was published also in [11].

The comparison of the refitted regression with original [9] can be seen from figure A1.

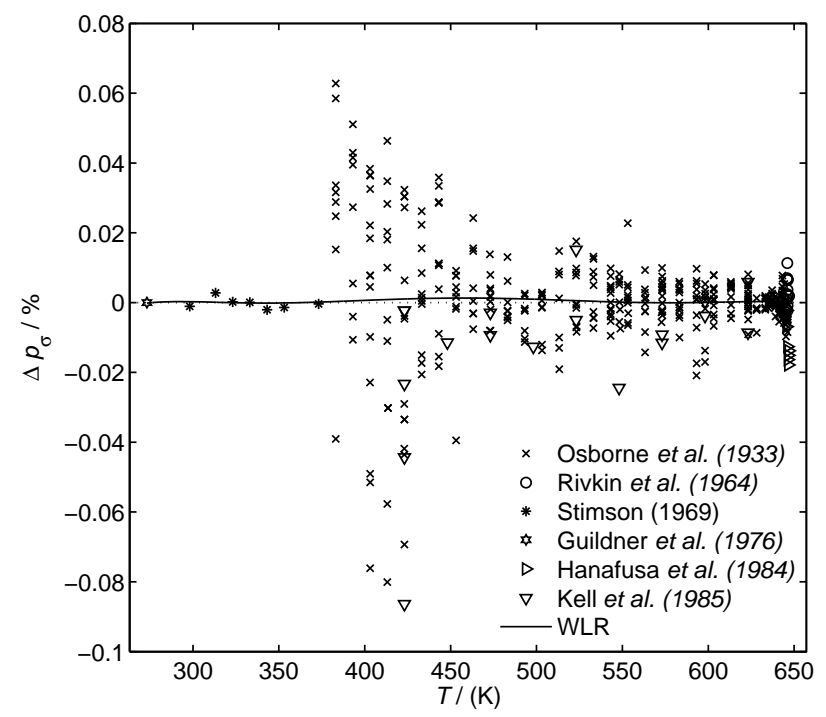

Fig. A1. Percentage deviation of saturated pressure from [9] regression.

A confidence interval along to saturation vapour pressure derivative function can be determined for selected temperatures $T^{*}$ as

$$
\frac{\mathrm{d} p_{\mathrm{s}}}{\mathrm{d} T}\left(T^{*}\right) \pm \sqrt{\chi_{\alpha}^{2} \mathbf{J}^{* \prime} \mathbf{V}(\mathbf{a}) \mathbf{J}^{*}}
$$

where $J_{i}^{*}=\frac{\partial \frac{\mathrm{d} p_{\mathrm{s}}}{\mathrm{d} T}\left(T^{*}, a\right)}{\partial a_{i}}$ and $\frac{\partial \frac{\mathrm{d} p_{\mathrm{s}}}{\mathrm{d} T}}{\partial a_{i}}=-\frac{p_{\mathrm{s}}}{T}\left[A_{i} \sum_{k=1}^{6} a_{k}\left(A_{k}+\alpha_{k} \vartheta^{\left(\alpha_{k}-1\right)}\right)+\left(A_{i}+\alpha_{i} \vartheta^{\left(\alpha_{i}-1\right)}\right)\right]$

where $A_{i}=\frac{T c}{T} \vartheta^{\alpha_{i}}$ and $\alpha$ are the exponents of the equation (A1).

Then the relative confidence interval defined by equation (A2) can be seen from figure A2

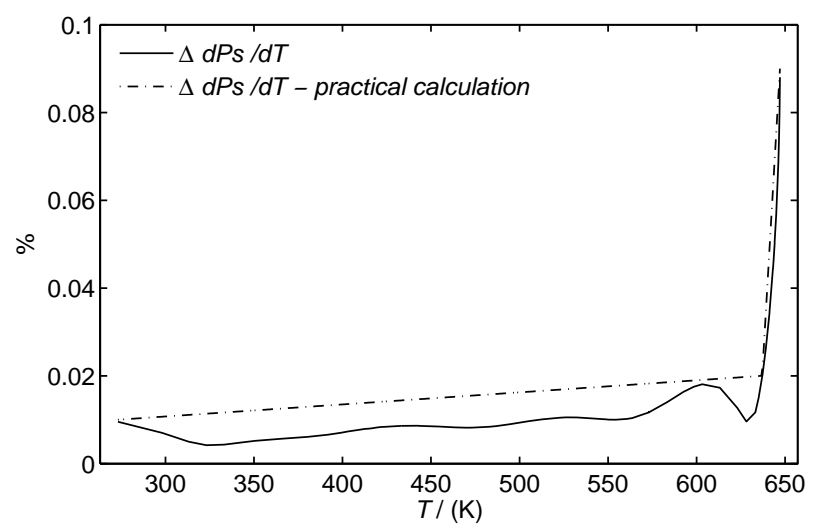

Fig. A2. Percentage uncertainty of saturated pressure derivation.

\section{A.2 The correction of the speed of sound for ignoring higher order virial coefficients}

The correction was calculated from equation

$$
\Delta w^{2}=w_{\text {IAPWS }}^{2}-w_{\text {virial }}^{2}
$$

where $w_{\text {IAPWS }}^{2}$ is the speed of sound calculated from IAPWS95 equation of state and $w_{\text {virial }}^{2}$ the speed of sound calculated from equation (11) without the correction and where the second virial coefficient $\mathrm{B}$ and its derivative are calculated from IAPWS95.

According to IAPWS95 the second virial coefficient is

$$
B=\frac{1}{\rho_{c}} \lim _{\delta \rightarrow 0} \phi_{\delta}^{r}(\delta, \tau)=\frac{\phi_{\delta}^{r}(0, \tau)}{\rho_{c}},
$$

where $\phi_{\delta}^{\mathrm{r}}$ is the derivation of residual Helmholtz energy with respect to $\delta=\rho / \rho_{c}$ and $\tau=T / T_{c}$.

Then the derivatives of $B$ are

$$
\begin{gathered}
B_{t}=-\frac{T_{c}}{\rho_{c}} \frac{\phi_{\delta \tau}^{r}(0, \tau)}{T}, \\
B_{t t}=\frac{T_{c}}{\rho_{c}}\left(\phi_{\delta \tau \tau}^{r} \frac{T_{c}}{T^{4}}+\phi_{\delta \tau}^{r} \frac{2}{T^{3}}\right) .
\end{gathered}
$$

The $\phi_{\delta \tau \tau}^{\mathrm{r}}$ is not published in [4] and therefore it will be determined here: 


$$
\begin{aligned}
\phi_{\delta \tau \tau}^{r} & =\sum_{i=1}^{7} n_{i} d_{i} t_{i}\left(t_{i}-1\right) \delta^{d_{i}-1} \tau^{t_{1}-2} \\
& +\sum_{i=8}^{51} n_{i} t_{i}\left(t_{i}-1\right) \delta^{d_{i}-1} \tau^{t_{1}-2}\left(d_{i}-c_{i} \delta^{c_{i}}\right) e^{-\delta^{c_{i}}} \\
& +\sum_{i=52}^{54} n_{i} \delta^{d_{i}-1} e^{-\alpha_{i}\left(\delta-\varepsilon_{i}\right)^{2}-\beta_{i}\left(\tau-\gamma_{i}\right)^{2}}\left[d_{i}-2 \alpha_{i} \delta\left(\delta-\varepsilon_{i}\right)\right] \\
\times\left\{t_{i}\left(t_{i}-1\right) \tau^{t_{1}-2}-2 \beta_{i} \tau^{t_{i}}\left[1+\frac{2 t_{i}\left(\tau-\gamma_{i}\right)}{\tau}-2 \beta_{i}\left(\tau-\gamma_{i}\right)^{2}\right]\right\} & \\
& +\sum_{i=55}^{56} n_{i}\left[\Delta^{b_{i}}\left(\frac{\partial^{2} \Psi}{\partial \tau^{2}}+\delta \frac{\partial^{3} \Psi}{\partial \delta \partial \tau^{2}}\right)\right. \\
+ & \frac{\partial \Delta^{b_{i}}}{\partial \tau}\left(2 \frac{\partial \Psi}{\partial \tau}+2 \delta \frac{\partial^{2} \Psi}{\partial \delta \partial \tau}+\delta \frac{\partial^{2} \Psi}{\partial \tau^{2}}\right) \\
+ & \left.\frac{\partial^{2} \Delta^{b_{i}}}{\partial \tau^{2}}\left(\Psi+\delta \frac{\partial^{3} \Psi}{\partial \delta \partial \tau^{2}}\right)+\frac{\partial^{2} \Delta^{b_{i}}}{\partial \tau \partial \delta} 2 \delta \frac{\partial \Psi}{\partial \tau}+\frac{\partial^{3} \Delta^{b_{i}}}{\partial \tau^{2} \partial \delta} \delta \Psi\right]
\end{aligned}
$$

where

$$
\begin{gathered}
\frac{\partial^{3} \Psi}{\partial \delta \partial \tau^{2}}=-\left[2 D_{i}(\tau-1)^{2}-1\right] 2 D_{i} \Psi C_{i} 2(\delta-1) \\
\frac{\partial^{3} \Delta^{b_{i}}}{\partial \tau^{2} \partial \delta}=2 b_{i}\left(b_{i}-1\right) \Delta^{b_{i}-2}\left\{\frac{\partial \Delta}{\partial \delta}+4 \Theta \frac{A_{i}}{\beta_{i}}\left[(\delta-1)^{2}\right]^{1 /\left(2 \beta_{i}\right)-1}\right. \\
\left.\times(\delta-1)+2 \Theta^{2} \frac{b_{i}-2}{\Delta} \frac{\partial \Delta}{\partial \delta}\right\}
\end{gathered}
$$

